\title{
From Mechanisms to Clinical Trials: Maslinic Acid, a Promising Modern Anti-inflammatory Drug?
}

\section{Jiahui Liu, Lei Yu and Hua Li*}

Department of Oncology, The General Hospital of Western Theater Command, China

*Corresponding Author: Hua Li, Department of Oncology, The General Hospital of Western Theater Command, China.
Received: March 08, 2021

Published: May 06, 2021

(C) All rights are reserved by Hua Li., et al.

\begin{abstract}
Chronic inflammation plays an essential role in various severe diseases and has become a main cause of death. Anti-inflammation medications for prevention and treatment of inflammation-related diseases are urgent. Maslinic Acid (MA), a pentacyclic triterpene abundant in olives, has been on our plates for years, but its anti-inflammatory mechanism has been clarified clearly until the last five years. Subsequently, the anti-inflammatory effect of MA for inflammation-related diseases was verified in pre-clinical and clinical studies. The MA studies reported in PubMed, Web of Science, Google Scholar, Baidu Scholar, CNKI, Science Direct, Springer and Wiley databases or registered in clinical trial management platforms were searched with the following key terms: maslinic acid, inflammation, inflammatory disease, pharmacological activity. This review from the perspective of medicine combed the anti-inflammation molecular mechanisms of MA and assessed the recent clinical trials, revealing the potential therapeutic use of Maslinic Acid as a new drug for the prevention and treatment of chronic inflammation-related diseases. These results should encourage more pre-clinical and clinical studies with stringent design and more strong evidence to accelerate the drug development MA.
\end{abstract}

Keywords: Maslinic Acid; Chronic Inflammation; NF-кB

\section{Abbreviations}

AKT: Protein Kinase B; AREs: Antioxidant Response Elements; BA: Bredemolic Acid; BMI: Body Mass Index; CAIA: Collagen Antibody-induced Arthritis; COX-2: Cyclooxygenase 2; eNOS: Endothelial NOS; GM-CSF: Granulocyte-Macrophage Colony Stimulating Factor; HEAC: Human Aortic Endothelial Cells; hGIIA: Human Group IIA; HO-1: Heme Oxygenase-1; HUVEC: Human Umbilical Vein Endothelial Cells; HUVECs: Human Umbilical Vein Endothelial Cells; IFN $\gamma$ : Interferon $\gamma$; IFN- $\gamma$ : Interferon- $\gamma$; IKK: I I B Kinase; IL-1: Interleukin-1; IL-10: Interleukin-10; IL-12: Interleukin-12; IL-13: Interleukin-13; IL-1R: Interleukin-1 Receptor; IL-23: Interleukin-23; IL-4: Interleukin-4; IL-6: Interleukin-6; IL-6R: Interleukin-6 Receptor; iNOS: Inducible Nitric Oxide Synthase; IкB: Inhibitor of NF- $\kappa \mathrm{B}$; JAK: Janus Kinase; LPS: Lipopolysaccharide;
MA: Maslinic Acid; MAP3: Mitogen Activated Protein 3; MAPK: Mitogen-Activated Protein Kinase; NAFLD: Non-Alcoholic Fatty Liver Disease; NF- $\kappa$ B: Nuclear Factor- $\kappa B$; Nrf2: Nuclear Factor Erythroid 2-Related Factor 2; OA: Osteoarthritis; PI3K: Phosphatidylinositol 3 Kinase; PKB (also called Akt): Protein Kinase B; QOL: Quality of Life; RHD: Rel Homology Domain; ROI: reactive oxygen intermediates; ROS: Reactive Oxygen Species; sPLA2: Secretory Phospholipase A2; STAT: Signal Transducer and Activator of Transcription; STAT: Signal Transducer and Activator of Transcription; TAD: Carboxy-terminal Transactivation Domains; TGF- $\beta$ : Transforming Growth Factor $\beta$; TLR: Toll-like Receptors; TNF: Tumor Necrosis Factor; TNFR: Tumor Necrosis Factor Receptor; TPA: 12-0-tetradecanoylphorbol13-acetate; VOO: Virgin Olive Oil; VSMCs: Vascular Smooth Muscle Cells; WBVT: Whole-Body Vibration Training 


\section{Introduction}

Inflammation is a crucial biological process in maintaining homeostasis and repairing damaged tissue. Nevertheless, chronic inflammation plays a vital role in the development of many severe diseases, including rheumatoid arthritis, asthma, chronic inflammatory bowel diseases, type 2 diabetes, neurodegenerative diseases [1], cardiovascular disease [2] and cancer. With more than 50\% of all deaths being attributable to inflammatory disorders, antiinflammation treatment is mounting concerns and interest. Antiinflammation treatment could help suppress or alleviate disease symptoms such as seizures in epilepsies [3], inflammatory erosion, tissue hyperplasia [4], etc.

Maslinic acid (MA, 2- $\alpha$, 3- $\beta$-dihydroxiolean-12-en-28-oic acid), a natural compound abundant in olives, has been widely consumed in daily diet or in traditional medicinal herbs in Europe (Mediterranean region), Asia (China, Japan, Korea, India), South America (such as Brazil), etc. [5]. A wide range of its biological activities includes anti-inflammation, antioxidant [6], antitumor, antidiabetic [7], antimicrobial [8] and growth-stimulating effects, has been observed in various research models. In recent years, mounting evidence of MA's anti-inflammatory mechanism further confirmed its anti-inflammatory effect observed in traditional usage, suggesting its potential to be applied as anti-inflammatory medication for inflammatory-related diseases. Thus, we searched the articles in PubMed, Web of Science, Google Scholar, Baidu Scholar, CNKI, Science Direct, Springer, Wiley databases and clinical trials of MA registered in clinical trial management platforms with the following terms: maslinic acid, inflammation, inflammatory disease, pharmacological activity. Here, from the perspective of medicine, we provide an overview of the recent progress made regarding the anti-inflammation mechanism of MA and assessed the recent clinical trials, hoping more strong evidence of stringent clinical studies to confirm the anti-inflammatory effect of MA alone for prevention and treatment of chronic inflammation-related disease.

\section{Characteristics of MA}

As people discover the remarkable biological properties of MA, it gradually gains attention as an excellent pharmacologically active product. MA (2- $\alpha, 3-\beta$-dihydroxyolean-12-en-28-oic acid) is a pentacyclic triterpenoid with the olean-12-ene substituted by hydroxy groups at positions 2 and 3 and a carboxy group at position 28 (the $2 \alpha, 3 \beta$ stereoisomer) (Table 1 ). The molecular weight of MA (C30H4804) is $472.7 \mathrm{~g} / \mathrm{mol}$ and other chemical properties are listed in table 1 . Its melting point is $249 \sim 250^{\circ} \mathrm{C}$. It is sparingly soluble in aqueous buffers but soluble in organic solvents such as ethanol, DMSO, and dimethylformamide, with 0.5, 20 and $15 \mathrm{mg} /$ $\mathrm{ml}$ solubility, respectively. The water-soluble MA solution could be obtained by first dissolving it in organic solvents and then diluting the solution with aqueous buffer of choice. MA can be separated and purified from lots of natural sources, most notably pomace olive oil. Using a high-speed countercurrent chromatography, 271.6 mg MA with $86.7 \%$ purity can be isolated from $600 \mathrm{mg}$ olive pulp within only $2 \mathrm{hrs}$ [9]. Although MA has long been a component of our food, its pharmacokinetics in vivo has not been clearly detected until recently. Series of MA pharmacokinetic parameters in rats were detected $[10,11]$, confirming that MA is a safe component rapidly absorbed and widely distributed. Later, a randomized controlled trial of healthy adults found out that after ingesting $6 \mathrm{mg}$ $\mathrm{MA}$ in $30 \mathrm{ml}$ of olive oil at $2 \mathrm{~h}$, the peak plasma concentration of MA could reach $30 \mathrm{ng} / \mathrm{ml}$ [12]. These data laid the foundation for the clinical application of MA as a solo medicine.

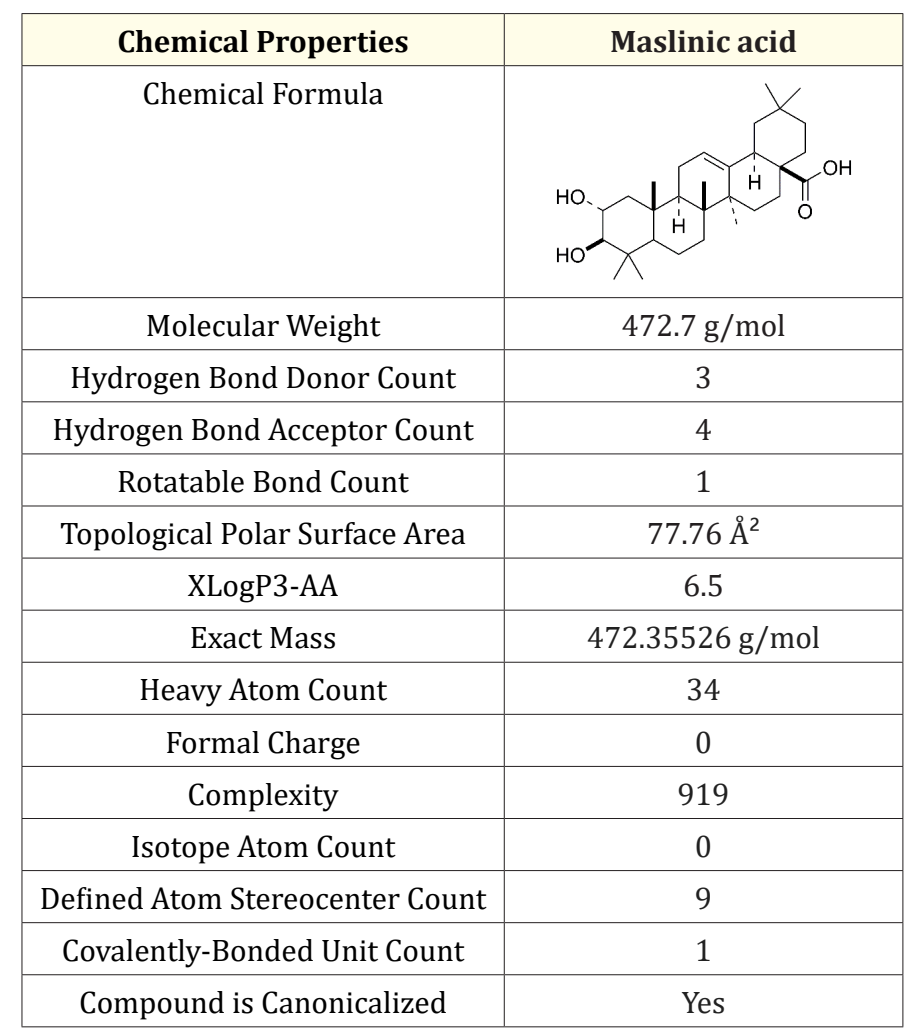

Table 1: Chemical properties of maslinic acid. 
Molecular mechanism of anti-inflammatory activity of MA

MA directly inhibits NF-кB activity via targeting ІкB

Inflammation is an innate immune response to harmful stimuli, including pathogens, toxic compounds, irradiation, damaged cells, and endogenous stress signals. These extracellular stimuli are mainly divided into two categories: the antigen receptors and the pattern-recognition receptors such as TLRs and cytokine receptors, including interleukin-1 receptor (IL-1R), interleukin-6 receptor (IL-6R) and tumor necrosis factor receptor (TNFR) to trigger intracellular inflammatory signaling pathways, most commonly the Nuclear Factor- $\kappa \mathrm{B}(\mathrm{NF}-\kappa \mathrm{B})$, the mitogen-activated protein kinase (MAPK) and Janus kinase (JAK)-signal transducer and activator of transcription (STAT) pathways.

$\mathrm{NF}-\kappa \mathrm{B}$ is a protein complex that controls the transcription of target genes, which regulate a wide variety of fundamental processes, including cell proliferation, differentiation, and survival. There are five proteins in the mammalian NF- $\mathrm{BB}$ family: NF- $\mathrm{BB} 1$ (also called p105/p50), NF-кB2 (also called p100/p52), RelA (also called p65),

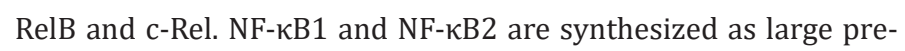
cursors, p105 and p100, then degraded by ubiquitin into the mature NF- $\mathrm{B}$ B subunits, p50 and p52, respectively. All the members have a Rel homology domain (RHD) in their N-terminus, a domain that is required for dimerization, DNA binding, and nuclear translocation. In addition, RelA (p65), RelB, and c-Rel contain carboxyterminal transactivation domains (TAD), necessary for the target gene transcription. p50 and p52, due to lack of TAD, need to bind with other members to from Rel/p50 and Rel/p52 heterodimers to trigger transcription. NF- $\kappa B$ dimers keep inactive "resting" state in the cytoplasm through binding with inhibitor of NF- $\kappa B$ (IкB) proteins. Once stimuli trigger the activation of the IкB, ІкB undergoes phosphorylation, ubiquitination, and degradation to release NF- $\kappa B$ dimers, thus NF- $\mathrm{KB}$ dimers turn to "active" state: translocating to the nucleus and binding specific DNA sequences to promote transcription of target genes including inflammatory and adhesion cytokines, prostaglandins, inducible nitric oxide synthase (iNOS) and cyclooxygenase 2 (COX-2). Therefore, NF- $\mathrm{BB}$ activation depends on I $\kappa$ degradation (Figure 1).

MA could not only prevent IкB- $\alpha$ phosphorylation to keep from IкB degradation but also upregulate the IкB- $\alpha$ expression [13], which, in turn, reduces NF-kB nuclear localization, phosphorylation and the DNA binding activity, finally inhibit inflammatory pathways [14] (Figure 1). The inhibition of inflammation via regulating IкB degradation by MA was further confirmed in the collagen

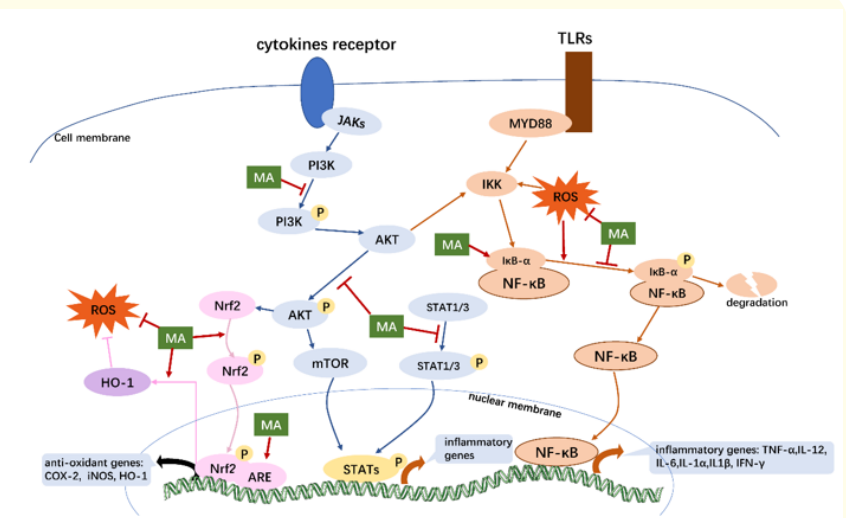

Figure 1: Scheme of the anti-inflammatory and antioxidant mechanisms of Maslinic acid. Brown lines and ellipses indicate NF- $\mathrm{BB}$ signal pathway. Blue lines and ellipses indicate PI3K/AKT and JAK/STAT pathways. Pink lines and ellipses indicate anti-oxidation-involved Nrf2/HO-1 pathway. Arrows denote activation or upregulation and T-shaped lines indicate inhibition. Abbreviations: TLR, Toll like receptor; MYD88, Myeloid differentiation pri-

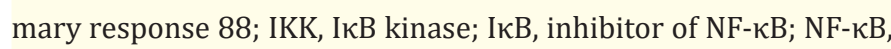
Nuclear Factor- $\kappa \mathrm{B}$; JAK, Janus kinase; PI3K, phosphatidylinositol 3-kinase; AKT, protein kinase B; mTOR, rapamycin; STAT, signal transducer and activator of transcription; Nrf2, nuclear factor erythroid 2-related factor 2; ARE, antioxidant response elements; HO1 , heme oxygenase-1; ROS, reactive oxygen species; TNF- $\alpha$, tumor necrosis factor- $\alpha$; IL-12, interleukin-12; IL-6, interleukin-6; IL-1 $\alpha$, interleukin- $1 \alpha$; IFN- $\gamma$, interferon- $\gamma$.

antibody-induced arthritis (CAIA) mice model: MA upregulated I $\mathrm{K}-\alpha$ expression to suppress the NF- $\kappa \mathrm{B}$ signaling pathway and to downregulate the proinflammatory cytokines IL-1 $\beta$, IL-6, interleukin-12 (IL-12) [15], tumor necrosis factor $\alpha$ (TNF- $\alpha$ ), IL1 $\beta$, and interferon $\gamma$ (IFN $\gamma)$. Thus, MA could alleviate carrageenan-induced paw edema and decreased the arthritis score of CAIA mice-fewer inflammatory cells in the knees of MA treated mice with no knee injury [16]. Mice models of other diseases verified this anti-inflammation molecular mechanism of MA. In the osteoarthritis mice model established by the medial meniscus, MA, in a dose-dependent manner, strongly inhibited p65 translocation and I $\mathrm{B} \alpha$ degradation in IL-1 $\beta$-stimulated cells [17]. In the LPS-induced acute liver injury mice model, MA attenuated NF- $\kappa \mathrm{B}$ activation by increasing the levels of phosphorylation of p65 and I $\mathrm{B}$ - $\alpha$ in liver tissues. MA ameliorated acute liver injury [18] and relieved arthritis [17] in the mouse model, suggesting it may use as a potential agent for the anti-inflammation treatment. 
MA indirectly inhibits NF- $\mathrm{KB}$ activity via the PI3K/AKT and JAK/STAT pathways

MA could inhibit other inflammatory signaling pathways such as the phosphatidylinositol 3-kinase (PI3K)/protein kinase B (AKT) [17] and JAK/STAT [19] to indirectly modulate NF- $\kappa B$ signaling (Figure 1). The PI3K/AKT pathway, an intracellular signal transduction pathway that promotes cell proliferation, cell survival, and growth, is the upstream pathway of NF- $\kappa B$. AKT activates the NF$\kappa B$ pathway through direct activation of I $\kappa B$ kinase (IKK). Yan-Lin Chen., et al. observed that MA could not only inhibit p65 translocation and I $\mathrm{B} \alpha \alpha$ degradation but also inhibit the phosphorylation of IL-1 $\beta$-induced PI3K and AKT at the same time. Consequently, MA decreases the expression of inflammatory mediators such as cyclooxygenase-2, IL-6, TNF- $\alpha$, and prostaglandin E2 in a concentrationdependent manner in human osteoarthritis (OA) chondrocyte. They further observed in the surgically induced OA mice model by medial meniscus that MA could ameliorated OA development via inhibition of AKT and PI3K phosphorylation, suggesting MA is a potential therapeutic agent against OA [17].

MA can also inhibit JAK/ signal transducer and activator of transcription (STAT) signaling (Figure 1), which is essential for signal transduction resulting from cytokine and growth factor receptors [20]. In the in-vitro model of human umbilical vein endothelial cells (HUVECs) treated with lipopolysaccharide (LPS), MA not only reduced NF- $\kappa$ B activity but also induced HO-1 expression to downregulate the STAT1 phosphorylation [19]. In the in-vivo mice skin tumor model treated by 12-0-tetradecanoylphorbol-13-acetate (TPA), MA significantly inhibited STAT3 phosphorylation on its tyrosine 705 and serine 727 residues and the downstream signaling of STAT3, resulting in reducing skin inflammation and inhibiting skin tumor promotion [21].

MA indirectly inhibits NF- $\mathrm{B}$ activity by activating Nrf2/HO-1 pathway to decrease ROS level

Besides anti-inflammatory effect, MA exerts anti-oxidation effect through the protein kinase B (Akt)/nuclear factor erythroid 2-related factor 2 (Nrf2)/heme oxygenase-1 (HO-1) signal pathway (Figure 1). Xiaofei., et al. found that MA induced Akt activation in a dose- and time-dependent manner, leading to an elevated expression of Nrf2 and HO-1 promoter, consequently increasing HO-1 expression in vascular smooth muscle cells (VSMCs) and protecting VMSCs from oxidative stress [22]. Ampofo, E., et al. found that MA induced Nrf2 transfer from cytoplasm to nucleus and increases the activity of antioxidant response elements (AREs) to activate the expression of HO-1 [23]. Another study observed that MA significantly increased the expression of antioxidant enzymes HO-1 and endothelial NOS (eNOS) and reduced oxidative DNA damage [24]. In addition, some evidence showed that MA could decreased the reactive oxygen species (ROS) level to show the antioxidant potential and improve cellular tolerance to oxidative stress. For example, MA could decrease the ROS level significantly in the impaired human aortic endothelial cells (HEAC) endothelial cell by high glucose, inhibited the inflammatory cytokines expression induced by high glucose and suppressed the cellular apoptosis ratio induced by high glucose [24]. Khalida Mokhtari., et al. found when B16F10 melanoma cells cultured without fetal bovine serum, Low concentration MA $(0.44 \mu \mathrm{M})$ could decreased ROS levels to improve tumor cell tolerance to oxidative stress [25]. In fact, ROS may activate $\mathrm{NF}-\kappa \mathrm{B}$ through alternative $\mathrm{I} \kappa \mathrm{B} \alpha$ phosphorylation, which result in the degradation of I $\mathrm{B} \alpha$ [26]. Therefore, MA decreases ROS level, also resulting in inhibition of the I $\kappa \mathrm{B} \alpha$ phosphorylation and NF- $\kappa \mathrm{B}$ activity, exerting anti-inflammatory effect [27]. The cardioprotective effect may be attributed to the ability of MA to ameliorate antioxidants [28].

\section{The effect of MA on inflammatory cells}

Inflammation, as a complex biological response to harmful stimuli, involves a variety of cells including neutrophils, macrophages, lymphocytes, and plasma cells. Macrophages play a central role in the development and maintenance of the inflammatory response. Macrophages have two distinct types, identified as M1 (or classically activated macrophages) and M2 (or selectively activated macrophages). M1 macrophages can be activated by lipopolysaccharide (LPS) or be induced by interferon- $\gamma$ (IFN- $\gamma$ ), TNF- $\alpha$, or granulocyte-macrophage colony stimulating factor (GM-CSF). M1 exerts pro-inflammatory response through secreting inflamma-

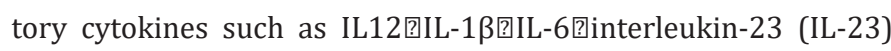
and TNF- $\alpha$ and producing inducible nitric oxide synthase (iNOS) and reactive oxygen intermediates (ROI). M2 macrophages can be induced and activated by interleukin-4 (IL-4), interleukin-13 (IL13), interleukin-10 (IL-10), transforming growth factor $\beta$ (TGF- $\beta$ ), glucocorticoids, immune complexes, etc. It has anti-inflammatory, angiogenesis, and tissue repair functions [29]. Macrophages constantly shift M1 or M2 functional phenotype to regulate tissue homeostasis. It has become a major target in the treatment of many 
diseases. Cristina., et al. found that MA could increase IFN- $\gamma$ production, which leads to M1 polarization and a decrease in IL-4 and IL-6 production. This then leads to M2 polarization in THP-1 (human acute monocytic leukemia) macrophages. Meanwhile, MA increased cytokines related to macrophages recruitment such as IL-8, IL-1 $\alpha$, IL-1 $\beta$, and pro-inflammatory cytokine IL-6 [30]. A recent study gave a similar conclusion with the effect of MA on THP-1 macrophages that MA increases IL- $1 \beta$ cytokines and inhibits TNF- $\alpha$ production in LPS-induced human U937 macrophage [31].

MA also acts on monocyte and neutrophil to inhibit inflammation. Yap, WH., et al. found that MA could bind with human group IIA (hGIIA)-secretory phospholipase A2 (sPLA2) enzyme to inhibit the access of catalytic calcium ion for enzymatic reaction and to block hGIIA-sPLA2 to bind with membrane phospholipid, leading to reduce hGIIA-sPLA2-induced THP-1 monocyte adhesive and migratory capabilities [32]. Subsequently, Yap, WH., et al. confirmed that MA could inhibit THP-1 monocyte adhesion to human umbilical vein endothelial cells (HUVEC) cells but induce trans-endothelial migration only at low concentration. Meanwhile, MA down-regulates both gene and protein expression on VCAM-1 and MCP-1 in HUVECs, thus to further reduce monocytes adhesion to endothelial cells [33]. Thiele., et al. found MA could reduce dermatitis edema caused by croton oil in mouse ears by reducing neutrophil infiltration [34].

\section{MA Clinical trials for inflammation-related diseases}

The NF- $\kappa$ B pathway is usually aberrantly activated in a sustained inflammatory response which contributes to the pathophysiology of many chronic diseases and the development of inflammation-associated cancer. Inhibition of the NF- $\mathrm{KB}$ signaling became a promising strategy for the treatment of type 2 diabetes, rheumatoid arthritis, metabolic syndrome, cardiovascular disease, ischemia/ reperfusion injury, and cancer. For example, metformin, a first-line medication of type 2 diabetes, ameliorates the proinflammatory state via downregulation of NF- $\mathrm{KB}$ signaling in type 2 diabetes and in cancer. Salsalate, an IкB kinase $\beta$-NF-kB inhibitor, could decrease $\mathrm{C}$-reactive protein and expediate blood glucose, and promote insulin secretion in diabetes clinical trials [35]. Preclinical rodent studies extended the application of this strategy to target NF- $\kappa B$ to other disease models. For example, NF- $\kappa \mathrm{B}$ downregulation could reduce ischemia/reperfusion brain injury and significantly reduce inflammation and infarct size, alleviating ischemic stroke [36]. By inhibiting the heme oxygenase-1(HO-1)/nuclear factor erythro- cyte 2-related factor 2(Nrf-2) pathway, NF- $\mathrm{B}$ activated could also significantly relieve inflammatory symptoms in rheumatoid arthritis murine model [37]. Strategies to target NF- $\mathrm{BB}$ pathways as such have led to some encouraging pre-clinical outcomes in cancer treatment [38]. IMD-0560, An IкB kinase $\beta$ Inhibitor that could hold $\mathrm{NF}-\kappa \mathrm{B}$ dimers in the "resting" state, showed potent therapeutic efficacy in the ovarian cancer xenograft mice model.

The anti-inflammatory and antioxidative properties of MA has been confirmed in a wide variety of animal disease models (Summarized in table 2). Besides MA, some derivatives of MA were investigated regarding their anti-inflammation properties in some in vivo inflammation models. Benzyl $(2 \alpha, 3 \beta)$ 2,3-diacetoxy-oleann12-en-28-amide (EM2), a benzylamide derivative of MA, also showed anti-inflammatory property in TPA-induced local acute inflammation model [39].

Following the encouraging results of these pre-clinical studies, clinical studies on the anti-inflammatory effects of maslinic acid are gradually carried out. The earliest clinical trial exploring the benefits of MA was on arthritis, a disease highly related with chronic inflammation. In 2016, Satoshi F., et al. performed a randomized, double-blind, placebo-controlled trial on 20 volunteers with mild knee joint pain in Japan to investigate the effects of consuming orally administered 50 mg MA daily for 12 weeks. The results showed that MA was most likely to improve joint condition and quality of life (QOL) by reducing pain and inflammatory response, resulting in significant decrease of the body weight and body mass index (BMI) [46]. Subsequently, they performed an open-label community residents-based clinical study on an isolated island with 29 elderly residents (mean $70.0 \pm 10.1$ years). They were given $30 \mathrm{mg}$ of MA daily for 16 weeks. The results showed that intake of MA could decrease knee joint pain (VAS and JKOM score), body weight, BMI, and could improve physical-related QOL [47]a conclusion in accordance with the previous trial. Later, another research team in Japan performed a double-blinded, placebo-controlled, randomized intervention study that enrolled women aged 65-85 years with knee osteoarthritis (OA). The results showed that whole-body vibration training (WBVT) in conjunction with ingesting three $50 \mathrm{mg}$ MA capsules (16.7 mg of MA) at breakfast for 20 weeks could reduce knee OA and remarkably improve knee and muscle function. This suggests the anti-inflammatory supplement MA is an effective treatment when used in combination with WBVT for improving knee muscle strength [48]. In 2018, a parallel, double-blind, randomized, placebo-controlled trial with 36 partici- 


\begin{tabular}{|c|c|c|c|c|}
\hline Chronic Disease & Animal Model & $\begin{array}{l}\text { Dose MA } \\
\text { (mg/kg) }\end{array}$ & Notable Changes Observed & Reference(s) \\
\hline \multirow[t]{2}{*}{ Arthritis } & $\begin{array}{l}\text { Collagen antibody induced in } \\
\text { arthritis DBA/1J mice }\end{array}$ & $200 \mathrm{mg} / \mathrm{kg}$ & $\begin{array}{c}\text { Reduced paw edema } \\
\text { Reduced TNF- } \alpha \text {, IL-1b levels } \\
\text { Inhibited TLR and NF- } \kappa \text { B signaling pathways } \\
\text { Upregulated gene expression of type III collagen } \\
\text { Inhibited leukotriene biosynthesis pathway }\end{array}$ & [15] \\
\hline & $\begin{array}{l}\text { Collagen antibody induced in } \\
\text { arthritis DBA/1J mice }\end{array}$ & $100 \mathrm{mg} / \mathrm{kg}$ & $\begin{array}{l}\text { Reduced the gene expression of TNF- } \alpha \text {, IL-1 } \beta \text {, and } \\
\text { IFN- } \gamma \\
\text { Decreased the paw arthritis score } \\
\text { Reduction in inflammatory cells } \\
\text { Reduced joint destruction }\end{array}$ & [16] \\
\hline $\begin{array}{l}\text { Ischemia/ } \\
\text { reperfusion }\end{array}$ & $\begin{array}{l}\text { I/R model of injury of blood } \\
\text { vessel in BALB/c mice }\end{array}$ & $20 \mathrm{mg} / \mathrm{kg}$ & $\begin{array}{l}\text { Increased the number of rolling leukocytes } \\
\text { Stabilize volumetric blood flow } \\
\text { Reduced leukocyte adherence during the early I/R }\end{array}$ & {$[23]$} \\
\hline Osteoarthritis & $\begin{array}{l}\text { DMM surgical induced in } \\
\text { C57BL/6 mice }\end{array}$ & $10 \mathrm{mg} / \mathrm{kg}$ & $\begin{array}{l}\text { Reversed hypocellularity } \\
\text { Reduced cartilage cauterization } \\
\text { Reduced proteoglycan degradation }\end{array}$ & {$[17]$} \\
\hline Sepsis & $\begin{array}{l}\text { Cecal ligation and puncture in- } \\
\text { duced sepsis in C57BL/6 mice }\end{array}$ & $0.7 \mathrm{mg} / \mathrm{kg}$ & $\begin{array}{l}\text { Improved the survival rate of mice } \\
\text { Reduced impairment of the lung tissue }\end{array}$ & [40] \\
\hline \multirow[t]{2}{*}{ Obesity } & $\begin{array}{l}\text { High fat diet induced obesity in } \\
\text { C57BL/6J mice }\end{array}$ & $38 \mathrm{mg} / \mathrm{kg}$ & $\begin{array}{l}\text { Improved insulin sensitivity and lipid homeostasis } \\
\text { Enhanced glucose tolerance } \\
\text { Reduction on body weight } \\
\text { Reduced pro-inflammatory genes in liver and adipose } \\
\text { tissue } \\
\text { Restored vascular function }\end{array}$ & {$[41]$} \\
\hline & $\begin{array}{l}\text { High fat diet induced obesity } \\
\text { C57BL/6 mice }\end{array}$ & $20 \mathrm{mg} / \mathrm{kg}$ & $\begin{array}{l}\text { Reduced liver weight and lipid accumulation } \\
\text { Improved hepatocyte steatosis } \\
\text { Reduced leptin, and free fatty acid concentrations }\end{array}$ & {$[42]$} \\
\hline Leukemia & $\begin{array}{l}\text { Injected(i.p.) WEHI-3 cells } \\
\text { induced leukemia in BALB/c } \\
\text { mice }\end{array}$ & $32 \mathrm{mg} / \mathrm{kg}$ & $\begin{array}{c}\text { Enhanced macrophage phagocytosis } \\
\text { Improved NK cell activities }\end{array}$ & [43] \\
\hline Seizure & $\begin{array}{c}\text { kainic acid induced seizure in } \\
\text { C57BL/6 mice }\end{array}$ & $40 \mathrm{mg} / \mathrm{kg}$ & $\begin{array}{l}\text { Improved the survival rate of mice } \\
\text { Reduced seizure scores } \\
\text { Reduced hippocampal inflammatory and apoptotic } \\
\text { injury } \\
\text { Alleviated oxidative injury }\end{array}$ & [44] \\
\hline
\end{tabular}




\begin{tabular}{|c|c|c|c|c|}
\hline $\begin{array}{l}\text { Myocardial infarc- } \\
\text { tion }\end{array}$ & $\begin{array}{l}\text { Isoprenaline induced in albino } \\
\text { wistar rat }\end{array}$ & $15 \mathrm{mg} / \mathrm{kg}$ & $\begin{array}{c}\text { Reduced heart edema } \\
\text { Reduced infiltrated inflammatory cells } \\
\text { NO necrosis of cardiac fibers } \\
\text { Preserved the structural integrity of myocardium } \\
\text { Attenuated the increase of tumors and lesions }\end{array}$ & {$[28]$} \\
\hline \multirow[t]{2}{*}{ Colon Tumor } & $\begin{array}{c}\text { Azoxymethane/dextran sulfate } \\
\text { sodium induced colon tumor in } \\
\text { C57BL/6J mice }\end{array}$ & $30 \mathrm{mg} / \mathrm{kg}$ & Reduced tumor volume, weight and number & \multirow[t]{2}{*}[45]{} \\
\hline & $\begin{array}{c}\text { HCT116 xenograft tumor } \\
\text { induced colon tumor in BALB/c } \\
\text { nude mice }\end{array}$ & $30 \mathrm{mg} / \mathrm{kg}$ & Reduction in inflammatory cytokines & \\
\hline Liver injury & $\begin{array}{l}\text { LPS/D-gal induced acute liver } \\
\text { injury in BALB/c mice }\end{array}$ & $50 \mathrm{mg} / \mathrm{kg}$ & $\begin{array}{l}\text { Attenuated liver histopathologic changes } \\
\text { Reduced inflammatory cytokines in serum and liver } \\
\text { tissues } \\
\text { Attenuated oxidative stress } \\
\text { Reduced neutrophil infiltration }\end{array}$ & {$[18]$} \\
\hline Pulmonary injury & LPS induced in C57BL/6 mice & $0.7 \mathrm{mg} / \mathrm{kg}$ & $\begin{array}{l}\text { Suppressed the levels of TNF- } \alpha \text { and iNOS } \\
\text { Alleviated lung tissue injury }\end{array}$ & [19] \\
\hline
\end{tabular}

Table 2: Overview of animal models used to study the anti-inflammatory and anti-oxidation properties of MA. Note: DMM: Destabilization of the medial meniscus, I/R: Ischemia/reperfusion, LPS: Lipopolysaccharide, i.p.: Intraperitoneally, D-gal: D-galactosamine.

pants in Japan came to the same conclusion that MA combined with resistance training could significantly improve muscle mass and grip strength, and reduce knee pain [49]. Although these clinical studies had limited statistical power with small sample size, they evaluated the anti-inflammatory effect of MA with randomized controlled trial design. More clinical studies of MA treatment with large sample size and even for other inflammation-related diseases are still needed in the future.

In addition to treatment of inflammation-related diseases, daily supplement of MA for health benefits has been tried to evaluate in clinical trials. A randomized, double-blind, crossover, controlled study with 54 healthy volunteers (aged 20 to 50) was carried out to compare three kinds of olive oil consumption: virgin olive oil (VOO), OVOO (VOO with a high phenolic content), and FOO (OVOO with oleanolic and maslinic acids) for coronary heart disease (ClinicalTrials.gov ID: NCT02520739) [50]. Three years later, the results of this NUTRAOLEUM Study become a first-level evidence on the in vivo health benefits of olive oil triterpenes (oleanolic and maslinic acids) in healthy human-decreasing DNA oxidation via CoQ9 and CoQ10 and plasma inflammatory biomarkers such as IL-8 and TNF- $\alpha$ after three weeks of supplementation [6]. From this study, we confirmed the health benefits of the consumption of oleanolic and maslinic acids, but the benefits of MA alone still need to be confirmed by other clinical trials with rigorous design.

\section{Conclusion}

Inflammation is involved in various severe diseases and is recognized as a deadly symptom that is becoming increasingly prevalent. To inhibit the inflammation becomes necessary. MA is a natural anti-inflammatory ingredient in the Mediterranean diet and in the Traditional Chinese Medicine. As the mechanisms underlying the anti-inflammatory and antioxidant role of MA has gradually been elucidated and more clinical trials data would be obtained, the application of MA in the prevention and treatment of chronic inflammation-related diseases would become more and more extensive in the future. 


\section{Bibliography}

1. J Sabatino., et al. "B cells in autoimmune and neurodegenerative central nervous system diseases". Nature Reviews Neuroscience 20.12 (2019): 728-745.

2. J Li., et al. "Dietary Inflammatory Potential and Risk of Cardiovascular Disease Among Men and Women in the U.S". Journal of the American College of Cardiology 76.19 (2020): 2181-2193.

3. JA French., et al. "Clinical studies and anti-inflammatory mechanisms of treatments". Epilepsia 58 (2017): 69-82.

4. H He., et al. "Mild atopic dermatitis lacks systemic inflammation and shows reduced nonlesional skin abnormalities". The Journal of Allergy and Clinical Immunology (2020).

5. A Rajopadhye and AS Upadhye. "Estimation of Bioactive Compound, Maslinic Acid by HPTLC, and Evaluation of Hepatoprotective Activity on Fruit Pulp of Ziziphus jujuba Mill. Cultivars in India". Evidence-Based Complementary and Alternative Medicine 2016 (2016): 4758734.

6. E Sanchez-Rodriguez., et al. "Effects of Virgin Olive Oils Differing in Their Bioactive Compound Contents on Biomarkers of Oxidative Stress and Inflammation in Healthy Adults: A Randomized Double-Blind Controlled Trial". Nutrients 11.3 (2019).

7. BN Mkhwanazi., et al. "Triterpene derivative improves the renal function of streptozotocin-induced diabetic rats: a followup study on maslinic acid". Renal Failure 41.1 (2019): 547-554.

8. CM Martin-Navarro., et al. "Amoebicidal Activity of Caffeine and Maslinic Acid by the Induction of Programmed Cell Death in Acanthamoeba". Antimicrobe Agents Chemotherapy 61.6 (2017).

9. X Huang., et al. "Continuous separation of maslinic and oleanolic acids from olive pulp by high-speed countercurrent chromatography with elution-extrusion mode". Journal of Separation Science 42.11 (2019): 2080-2088.

10. G Lozano-Mena., et al. "Identification of gut-derived metabolites of maslinic acid, a bioactive compound from Olea europaea L". Molecular Nutrition and Food Research 60.9 (2016): 2053-2064.
11. M Sanchez-Gonzalez., et al. "Population pharmacokinetics of maslinic acid, a triterpene from olives, after intravenous and oral administration in rats". Molecular Nutrition and Food Research 58.10 (2014): 1970-1979.

12. R de la Torre., et al. "Pharmacokinetics of maslinic and oleanolic acids from olive oil - Effects on endothelial function in healthy adults. A randomized, controlled, dose-response study". Food Chemistry 322 (2020): 126676.

13. K Shimazu., et al. "The Anti-Arthritis Effect of Olive-Derived Maslinic Acid in Mice is Due to its Promotion of Tissue Formation and its Anti-Inflammatory Effects". Molecular Nutrition and Food Research 63.3 (2019): e1800543.

14. E Ampofo., et al. "Maslinic acid alleviates ischemia/reperfusion-induced inflammation by downregulation of NFKBmediated adhesion molecule expression". Molecular Nutrition and Food Research 9.1 (2019): 6119.

15. K Shimazu., et al. "The Anti-Arthritis Effect of Olive-Derived Maslinic Acid in Mice is Due to its Promotion of Tissue Formation and its Anti-Inflammatory Effects". Molecular Nutrition and Food Research 63.3 (2019): e1800543.

16. S Fukumitsu., et al. "Anti-inflammatory and anti-arthritic effects of pentacyclic triterpenoids maslinic acid through NFкB inactivation". Molecular Nutrition and Food Research 60.2 (2016): 399-409.

17. Y Chen., et al. "Maslinic acid prevents IL-1 $\beta$-induced inflammatory response in osteoarthritis via PI3K/AKT/NF- $\kappa B$ pathways". Journal of Cellular Physiology 236.3 (2020): 1939-1949.

18. Yy Wang., et al. "Maslinic acid protects against lipopolysaccharide/D-galactosamine-induced acute liver injury in mice". Microbial Pathogenesis 119 (2018): 49-53.

19. W Lee., et al. "Maslinic Acid Ameliorates Inflammation via the Downregulation of NF- $\kappa B$ and STAT-1". 9.2 (2020): 106.

20. J Darnell., et al. "Jak-STAT pathways and transcriptional activation in response to IFNs and other extracellular signaling proteins". Science (New York, N.Y.) 264.5164 (1994): 1415-1421.

21. J Cho., et al. "Evaluation of pentacyclic triterpenes found in Perilla frutescens for inhibition of skin tumor promotion by 12-0-tetradecanoylphorbol-13-acetate". Oncotarget 6.36 (2015): 39292-306. 
22. X Qin., et al. "Maslinic acid protects vascular smooth muscle cells from oxidative stress through Akt/Nrf2/HO-1 pathway". Molecular and Cellular Biochemistry 390 (2014): 61-67.

23. E Ampofo., et al. "Maslinic acid alleviates ischemia/reperfusion-induced inflammation by downregulation of NFkBmediated adhesion molecule expression". Scientific Reports 9.1 (2019): 6119.

24. F Li., et al. "Maslinic acid inhibits impairment of endothelial functions induced by high glucose in HAEC cells through improving insulin signaling and oxidative stress". Biomedicine and pharmacotherapy $=$ Biomedecine and pharmacotherapie 95 (2017): 904-913.

25. K Mokhtari., et al. "Maslinic Acid, a Triterpene from Olive, Affects the Antioxidant and Mitochondrial Status of B16F10 Melanoma Cells Grown under Stressful Conditions". EvidenceBased Complementary and Alternative Medicine 2015 (2015): 272457.

26. MJ Morgan., et al. "Crosstalk of reactive oxygen species and NF|kappa.|B signaling”. Cell Research 21 (2011): 103-115.

27. Y Yang., et al. "Maslinic Acid Protected PC12 Cells Differentiated by Nerve Growth Factor against $\beta$-Amyloid-Induced Apoptosis". Journal of Agricultural and Food Chemistry 63.47 (2015): 10243-10249.

28. AHS A., et al. "Maslinic acid ameliorate electrolytes, membrane bound ATPases, antioxidants and histopathology in isoprenaline attenuated myocardial toxicity in rats". Journal of King Saud University - Science 32.1 (2020): 1055-1059.

29. A Shapouri-Moghaddam., et al. "Macrophage plasticity, polarization, and function in health and disease". Journal of Cellular Physiology 233.9 (2018): 6425-6440.

30. C Sánchez-Quesada., et al. "Maslinic Acid enhances signals for the recruitment of macrophages and their differentiation to $\mathrm{m} 1$ state". Evidence-Based Complementary and Alternative Medicine : eCAM 2015 (2015): 654721.

31. H Wee., et al. "Effects of Vitex trifolia L. leaf extracts and phytoconstituents on cytokine production in human U937 macrophages". BMC Complementary Medicine and Therapies 20.1 (2020): 91.
32. W Yap., et al. "Inhibition of Human Group IIA-Secreted Phospholipase A2 and THP-1 Monocyte Recruitment by Maslinic Acid". Lipids 51.10 (2016): 1153-1159.

33. S Phang., et al. "Maslinic acid suppresses macrophage foam cells formation: Regulation of monocyte recruitment and macrophage lipids homeostasis". Vascular Pharmacology (2020): 106675.

34. T de Brum., et al. "Ethnopharmacological study and topical anti-inflammatory activity of crude extract from Poikilacanthus glandulosus (Nees): Ariza leaves". Journal of Ethnopharmacology 193 (2016): 60-67.

35. N Esser., et al. "Anti-inflammatory agents to treat or prevent type 2 diabetes, metabolic syndrome and cardiovascular disease". Expert Opinion on Investigational Drugs 24.3 (2015): 283-307.

36. JA Howell and GL Bidwell. "Targeting the NF- $\kappa B$ pathway for therapy of ischemic stroke". Therapeutic Delivery 11.2 (2020): 113-123.

37. F Zhang., et al. " $\beta$-Sitosterol-loaded solid lipid nanoparticles ameliorate complete Freund's adjuvant-induced arthritis in rats: involvement of NF- $\kappa B$ and HO-1/Nrf-2 pathway". Drug Delivery 27.1 (2020): 1329-1341.

38. R Freitas and CAM Fraga. "NF-kappaB-IKKbeta Pathway as a Target for Drug Development: Realities, Challenges and Perspectives". Current Drug Targets 19.16 (2018): 1933-1942.

39. I Pavel., et al. "Assessment of the Antiangiogenic and AntiInflammatory Properties of a Maslinic Acid Derivative and its Potentiation using Zinc Chloride". International Journal of Molecular Sciences 20.11 (2019).

40. W Lee., et al. "Inhibitory functions of maslinic acid, a natural triterpene, on HMGB1-mediated septic responses". Phytomedicine: International Journal of Phytotherapy and Phytopharmacology 69 (2020): 153200.

41. C Claro-Cala., et al. "Pomace Olive Oil Concentrated in Triterpenic Acids Restores Vascular Function, Glucose Tolerance and Obesity Progression in Mice". Nutrients 12.2 (2020). 
42. C Liou., et al. "Maslinic acid protects against obesity-induced nonalcoholic fatty liver disease in mice through regulation of the Sirt1/AMPK signaling pathway". FASEB Journal : official publication of the Federation of American Societies for Experimental Biology 33.11 (2019): 11791-11803.

43. KC Lai., et al. "Maslinic Acid Enhances Immune Responses in Leukemic Mice Through Macrophage Phagocytosis and Natural Killer Cell Activities In Vivo". In Vivo 33.1 (2019): 65-73.

44. Z Wang., et al. "Asiatic acid and maslinic acid attenuated kainic acid-induced seizure through decreasing hippocampal inflammatory and oxidative stress". Epilepsy Research 139 (2018): 28-34.

45. Q Wei., et al. "Maslinic Acid Inhibits Colon Tumorigenesis by the AMPK-mTOR Signaling Pathway". Journal of Agricultural and Food Chemistry 67.15 (2019): 4259-4272.

46. S Fukumitsu., et al. "Maslinic acid in olive fruit alleviates mild knee joint pain and improves quality of life by promoting weight loss in the elderly". Journal of Clinical Biochemistry and Nutrition 59.3 (2016): 220-225.

47. S Fukumitsu., et al. "Maslinic acid improves quality of life by alleviating joint knee pain in the elderly: results from a community-based pilot study". Journal of Clinical Biochemistry and Nutrition 61.1 (2017): 67-73.

48. J Yoon., et al. "Evaluation of maslinic acid with whole-body vibration training in elderly women with knee osteoarthritis". PLoS One 13.3 (2018): e0194572.

49. N Nagai., et al. "Maslinic acid derived from olive fruit in combination with resistance training improves muscle mass and mobility functions in the elderly". Journal of Clinical Biochemistry and Nutrition 64.3 (2019): 224-230.

50. S Biel., et al. "The NUTRAOLEOUM Study, a randomized controlled trial, for achieving nutritional added value for olive oils". BMC Complementary and Alternative Medicine 16.1 (2016): 404.

Volume 5 Issue 6 June 2021

(C) All rights are reserved by Hua Li., et al. 